Original Paper

\title{
Long Noncoding RNA H19/miR-675 Axis Promotes Gastric Cancer via FADD/Caspase 8/Caspase 3 Signaling Pathway
}

\author{
Jin Yan ${ }^{a, b}$ Yifeng Zhang ${ }^{a, b}$ Qiang She ${ }^{b, c}$ Xuan Li ${ }^{a, b}$ Lei Peng ${ }^{a, b}$ Xiaoyong Wang ${ }^{c, d}$ \\ Shiyu Liu ${ }^{b, e}$ Xiaoran Shen ${ }^{a, b}$ Weifeng Zhang ${ }^{a, b}$ Yu Donga,b Jieyu Lu ${ }^{\mathrm{b}}$ \\ Guoxin Zhanga,b \\ aDepartment of Gastroenterology, The First Affiliated Hospital with Nanjing Medical University, \\ Nanjing, ${ }^{b}$ The First Clinical Medical College, Nanjing Medical University, Nanjing, 'Department of \\ Gastroenterology, The First Affiliated Hospital of Yangzhou University, Yangzhou University, Yangzhou, \\ dDepartment of Gastroenterology, the No, 2 Hospital of Changzhou, Changzhou, eDepartment of \\ Gastroenterology, the No, 1 People's Hospital of Xuzhou, Xuzhou, China
}

\section{Key Words}

Gastric cancer • H19 • miR-675 • FADD • Caspase $8 \cdot$ Caspase 3

\begin{abstract}
Background: Long noncoding RNA (IncRNA) H19 is emerging as a vital regulatory molecule in the progression of different types of cancer and miR-675 is reported to be embedded in $\mathrm{H} 19$ 's first exon. However, their function and specific mechanisms of action have not been fully elucidated. The aim of this study was to identify a novel IncRNA-microRNA-mRNA functional network in gastric cancer. Methods: Quantitative real-time polymerase chain reaction (qRTPCR) was used to assess the relative expression of $\mathrm{H} 19$ and miR-675 in normal (GES-1) and gastric cancer cell lines (SGC-7901, SGC-7901/DDP) as well as in tumor tissues. Gain and loss of function approaches were carried out to investigate the potential roles of H19/miR-675 in cell proliferation and apoptosis. Moreover, Fas associated via death domain (FADD) was validated to be the target of miR-675 via luciferase reporter assay. Western blotting was used to evaluate the protein expression of related signaling pathway. Results: In our study H19 and miR-675 were increased in gastric cancer cell lines and tissues. Overexpression of H19 and miR-675 promoted cell proliferation and inhibited cell apoptosis, whereas knockdown of $\mathrm{H} 19$ and miR-675 inhibited these effects. By further examining the underlying mechanism, we showed that H19/miR-675 axis inhibited expression of FADD. FADD downregulation subsequently inhibited the caspase cleavage cascades including caspase 8 and caspase 3 . Conclusion: Taken together, our results point to a novel regulatory pathway H19/miR-675/ FADD/caspase 8/caspase 3 in gastric cancer which may be potential target for cancer therapy. (C) 2017 The Author(s)

J. Yan, Y. Zhang and Q. She are equal contributors.




\section{Introduction}

Gastric cancer is ranked the fourth most prevalent cancer, it is also one of the leading causes of cancer related deaths worldwide and thus it represents a major health burden [1]. Its formation is a multistep process which involves an interplay of many different genes in the tumor itself as well as in its surroundings [2]. Unfortunately there are no typical signs of gastric cancer until it's advanced stage. Although the progressive diagnose methods and standard chemotherapy protocols have been established, prognosis of gastric cancer patients is still very poor [3]. Thus, there is still a pressing need to elucidate the molecular mechanisms underlying gastric cancer progression as well as to identify key biomarkers and develop effective targeted therapies.

LncRNAs are generally defined as RNA transcripts longer than 200 nucleotides without protein coding function. LncRNAs have been shown to influence a number of cellular functions, such as cell proliferation, migration, invasion, and apoptosis, by modulating gene expression at transcriptional and posttranscriptional levels [4-7]. Recently, Zhao et al have performed a lncRNA microarray analysis of gastric tumors and found 3141 lncRNAs which were significantly differentially expressed in tumor tissue compared to the paired normal gastric tissue [8]. It has been reported that $\mathrm{H} 19$, a 2.3-kb lncRNA which is transcribed from H19/Igf2 gene cluster located on human chromosome 11p15.5, is among the strongly upregulated lncRNAs $[9,10]$. Furthermore, $\mathrm{H} 19$ has been reported to regulate tumorigenesis in recent years but whether $\mathrm{H} 19$ acts as a tumor suppressor or as a tumor promotor is still controversial $[11,12]$. In addition, the precise underlying mechanism of its function still remains elusive and needs to be further explored.

It is known that lncRNAs sometimes acquire function by acting as precursors of miRNAs, which post-transcriptionally regulate target genes through base pairing with specific sequences in 3'untranslated regions (UTRs) [13, 14]. H19 is reported as a precursor of primary miR-675 and generates it in the Drosha and Dicer splicing-dependent manner [15]. MiR-675 in turn has been reported to promote cancer by repressing tumor suppressor genes in a classical miRNA manner, but its growth restriction role in embryonic development has also been described $[16,17]$. However, the role of H19/miR-675 axis in tumorigenesis remains elusive and it has not been validated in animal models. Consequently, we speculated that H19 induced tumorigenesis may also be mediated by miR- 675 .

In this study, we found that the expression of both $\mathrm{H} 19$ and miR-675 were significantly increased in gastric cancer cells and tissues. Furthermore, we demonstrated that H19 promoted gastric cancer formation at least partially mediated by miR- 675 both in vivo an in vitro. Moreover, our results identified for the first time that FADD, a significant gene regulating cell apoptosis, is a direct target of miR-675. These results increase our understanding on the role of the IncRNA-miRNA-mRNA functional network in gastric cancer.

\section{Materials and Methods}

\section{Human tissue samples}

Paired gastric cancer tissue samples and corresponding adjacent noncancerous gastric samples of thirty-four patients were collected from the First Affiliated Hospital of Nanjing Medical University (Nanjing, China). All samples were confirmed as gastric cancer by pathological analysis and none of the patients had received chemotherapy or radiotherapy before surgical resection. Informed consent was obtained from all patients and this study was approved by the Ethical Committee of the First Affiliated Hospital of Nanjing Medical University.

\section{Cell lines and cell culture}

Human gastric cancer cell line SGC-7901, cisplatin resistant cell line SGC-7901/DDP and normal gastric epithelial cell line GES-1 were purchased from the Shanghai Institute of Cell Biology (Shanghai, China). Cells were cultured in PRIM-1640 (GIBCO, Rockville, USA) supplemented with 10\% fetal bovine serum (GIBCO, Rockville, USA) and $1 \%$ antibiotics and grown at $37{ }^{\circ} \mathrm{C}$ in humidified air with $5 \% \mathrm{CO}_{2}$. 


\section{Cellular Physiology Cell Physiol Biochem 2017;42:2364-2376 \begin{tabular}{ll|l} 
and Biochemistry Published online: August 18, 2017 & $\begin{array}{l}\text { (c) } 2017 \text { The Author(s). Published by S. Karger AG, Basel } \\
\text { www.karger.com/cpb }\end{array}$
\end{tabular}}

Yan et al.: H19/miR-675 Promote Gastric Cancer via FADD

\section{RNA extraction and $q R T-P C R$}

Total RNA from tissue and cell samples was extracted using a Trizol reagent (Takara, Otsu, Japan) following the manufacturers' instruction. For mRNA expression analysis, reverse transcription was carried out using reverse transcription kit (Takara, Otsu, Japan). For the miRNA expression analysis First-Strand cDNA Synthesis Kit (GeneCopoeia, Guangzhou, China) was used in reverse transcription. Real-time PCR was performed in triplicate using the SYBR Green PCR Kit (Takara). All reactions were performed on Applied Biosystems Step One Plus Real-Time PCR System (Applied Biosystems, Foster city, USA). Expression of GAPDH (for mRNA) and U6 (for miRNA) were used as endogenous controls. Comparative quantification was determined using the $2^{-\Delta \Delta C T}$ method. The following gene-specific oligonucleotides were used for qRTPCR experiments:

H19 forward: ACCACTGCACTACCTGACTC

reverse: CCGCAGGGGGTGGCCATGAA

FADD forward: GCTGGCTCGTCAGCTCAAA

reverse: ACTGTTGCGTTCTCCTTCTCT

GAPDH forward: CTGGGCTACACTGAGCACC

reverse: AAGTGGTCGTTGAGGGCAATG

miR-675 forward: CCCAGGGTCTGGTGCGGAGA

reverse: CAGGGGCTGAGCGGTGAGGG

U6 forward: CTCGCTTCGGCAGCACA

reverse: ACGCTTCACGAATTTGCGT

\section{Cell transfection}

For the cell transfection experiments, plasmid-H19, plasmid-FADD, plasmid-control, si-H19, si-FADD, and si-control RNA were synthesized by Invitrogen (Nanjing, China). MiR-675 mimic, miR-675 inhibitor, and their respective controls were bought from Genepharma company (Shanghai, China). Plasmid and oligonucleotide transfection were performed by using Lipofectamine 2000 reagent (Life Technologies Corporation, Carlsbad, USA) according to the manufacturers' instruction. Finally, $48 \mathrm{~h}$ or $72 \mathrm{~h}$ after transfection, transfected cells were collected and used in further experiments.

\section{Dual luciferase reporter assay}

For the dual luciferase reporter assay cDNA fragments from FADD containing the miR-675 binding sites were amplified by PCR and cloned into a pGL3 vector (Promega, Madison, USA). Next, cells were grown in the 24-well plate for 24 hours and were transfected with miR-675 mimic, miR-675 inhibitor, or respective controls, as well as luciferase reporters using Lipofectamine 2000 according to the manufacturers' protocol. Cells were harvested $48 \mathrm{~h}$ later, and the firefly and renilla luciferase activities were measured with Promega Glomax 2020 Single Tube Luminometer instrument (Promega). The light intensity from firefly luciferase was normalized with renilla luciferase signal. Each experiment was performed in triplicate.

\section{Western blot analysis}

For the western blot analysis total proteins were extracted with RIPA buffer and boiled for 5 min at $95{ }^{\circ} \mathrm{C}$. Next, protein lysates were separated by sodium dodecyl sulfate-polyacrylamide gel electrophoresis (SDS-PAGE) and proteins were transferred onto PVDF membranes (Millipore, Billerica, USA). Membranes were then blocked with $5 \%$ bovine serum albumin (BSA) for $2 \mathrm{~h}$ and were incubated with anti-humanFADD (Abcam, Cambridge, USA), pro-caspase 8, pro-caspase 3, actin (Cell Signaling Technology, Beverly, USA) at $4{ }^{\circ} \mathrm{C}$ overnight. After being washed with PBS-Tween-20 (PBS-T) for three times, membranes were incubated with secondary antibody for 1 hour at room temperature. After another three washes, the signal was measured using a chemiluminescent detection system (Pierce ECL Substrate Western blot detection system, Thermo, Rockford, IL, USA).

\section{Cell proliferation assay}

For the cell proliferation assay, after transfection, 1.0x104 cells per well were seeded into 96-well plates in RPMI-1640 containing 10\% FBS. Next, CCK-8 (Dojindo, Kumamoto, Japan) solution (10 $\mu$ l/well) was added and cells were incubated at $37^{\circ} \mathrm{C}$ in 5\% CO2 for 2 hours according to the manufacturers' instruction. Finally, the absorbance at $450 \mathrm{~nm}$ of each well was recorded using a microplate reader (Bio-Rad, USA). 


\section{Colony formation assay}

For the colony formation assay, cells were trypsinized and cultured on 6-well plates. After 10 days in culture, colonies were fixed in methanol for 20 minutes, stained with Crystal Violet (Sigma, St Louis, MO, USA).

\section{Apoptosis analysis}

For the analysis of apoptosis, Annexin V-FITC Apoptosis Detection Kit (Vazyme, Nanjing, China) was used to double stain cells according to the manufacturer s' protocol. In brief, FITC-Annexin V and PI were added and cells were incubated in dark for 30 minutes. Results were analyzed using the BD Biosciences FACS Calibur Flow Cytometer (BD Biasciences, Franklin Lakes, USA).

\section{Lentiviral infection and in vivo tumorigenicity assay}

Lentiviruses targeting H19 (LV-shH19), LV-miR675, and scramble control (LV-shNC) were obtained from GENE (Shanghai, China) and were used to stably infect SGC-7901 cells. All experimental procedures were conducted strictly in accordance with the Guide for the Care and Use of Laboratory Animals, and approved by the Nanjing Medical University Animal Studies Committee. All animals were housed at specific pathogen free condition. Stably infected cells were injected subcutaneously into 5-week-old male nude mice $\left(1 \times 10^{6}\right.$ cells $\left./ \mathrm{ml}, 0.2 \mathrm{ml}\right)$. Tumor growth was measured every four days after implantation, and the volume of each tumor was calculated was calculated using this formula: $V=($ length * width * width * $\pi) / 6$. Mice were euthanized 4 weeks after injection, and tumors were removed and weighed.

\section{Statistical analysis}

All data are described as the mean \pm s.d. Student's $t$ test was utilized to examine the statistical difference between groups. The correlation significance was determined by Spearman and Pearson correlation analyses. A two side $\mathrm{P}<0.05$ value was considered statistically significant. Statistical analyses were performed with the use of GraphPad Prim 5 (GraphPad Software, La Jolla, USA) or SPSS 20.0 (SPSS, Chicago, USA).

\section{Results}

FADD is a potential target of $H 19 / \mathrm{miR}-675$

It has been established that miR-675 derives from the first exon of H19 (Fig. 1A). In order to investigate whether $\mathrm{H} 19$ at least in part obtains its function from miR-675, we have examined their expression in gastric cancer line SGC-7901. For this purpose cells were transfected with pc-H19, pc-control, si-H19 or si-control. QRT-PCR analysis has shown sevenfold higher $\mathrm{H} 19$ expression in the pc-H19 transfected cells while an $80 \%$ decrease of H19 expression was detected in si-H19 transfected group compared with control cells. The miR-675 expression showed a trend similar to H19 expression with more than threefold upregulation in pc-H19 transfected cells and 70\% decrease in cells transfected with si-H19 compared to control group (Fig. 1B and C).

In order to elucidate the molecular mechanism of how miR-675-5p contributes to tumorigenesis, we searched the Targetscan database (www.targetscan.org) for possible miR675 targets. Among the predicted targets, FADD was chosen since it has a vital function in cell apoptosis. According to Targetscan, FADD contains a 10-mer seed miR-675 matches in its 3'UTR region. Furthermore, qPCR and western blot analysis performed in our study showed that FADD was downregulated in SGC-7901 cells transfected with miR-675 mimic while its expression was upregulated in cells in which miR-675 was inhibited. In addition, FADD was reduced in the presence of pc-H19 and increased in the presence of si-H19 compared to its expression in control cells (Fig. 1D, E, and F).

To confirm whether FADD was a direct target of miR-675, we cloned the sequence of FADD 3'UTR region with miR-675 predicted binding sites downstream of a luciferase reporter and co-transfected it into SGC-7901 cells with either miR-675 mimic, miR-675 inhibitor or respective scrambled controls (Fig. 1G). In our study luciferase levels were 


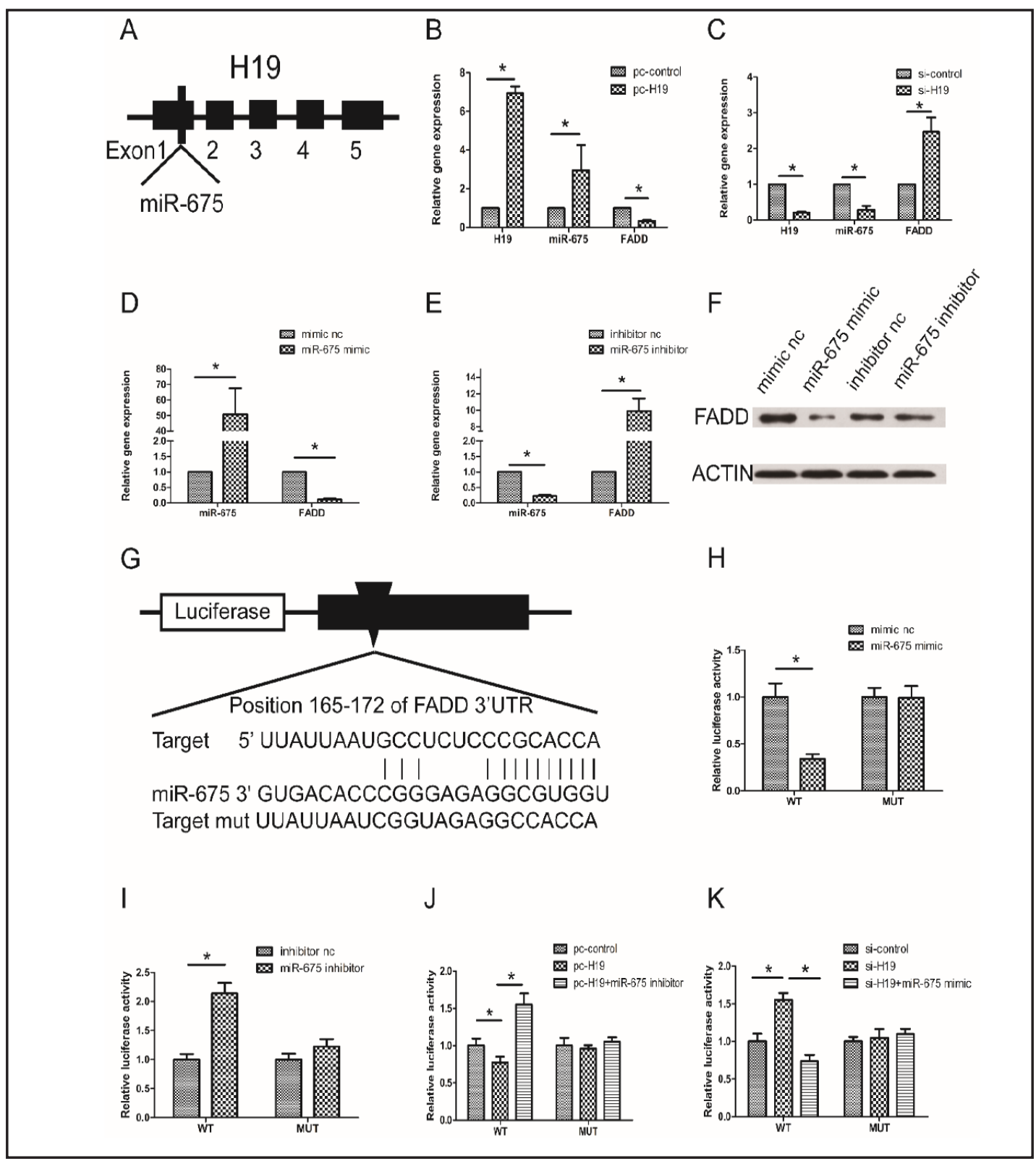

Fig. 1. FADD is a potential target of H19/miR-675 in SGC-7901 gastric caner cell line. (A) MiR-675 is encoded by exon 1 of H19. (B-C) H19, miR-675, and FADD expression were detected by qRT-PCR in gastric cancer cells after transfection with pc-H19, si-H19, and their respective controls. (D-E) MiR-675 and FADD mRNA expression were detected by qRT-PCR in gastric cancer cells transfected with miR-675 mimic, inhibitor or their respective controls. (F) Western blot analysis of FADD protein expression in gastric cancer cells transfected with miR-675 mimic, inhibitor or their respective controls; An unrelated protein Actin was used as the control. (G) Seed sequences of miR-675 matching the 3'UTR region of FADD; wild type and mutant FADD luciferase reporter plasmids were constructed. (H-K) Firefly luciferase activity in gastric cancer cells after their co-transfection with luciferase reporter construct and miR-675 mimic or miR-675 inhibitor, or pc-H19 or si-H19. Data are presented as the mean \pm SD of three independent experiments. *p $<0.05$.

reduced by more than $60 \%$ in the presence of miR- 675 mimic and increased to more than double in the presence of miR-675 inhibitor in comparison to control cells. Furthermore, this effect was reversed when miR-675 binding sites were mutated (Fig.1H and I). Thus we can conclude that FADD is a potential target of miR- 675. 


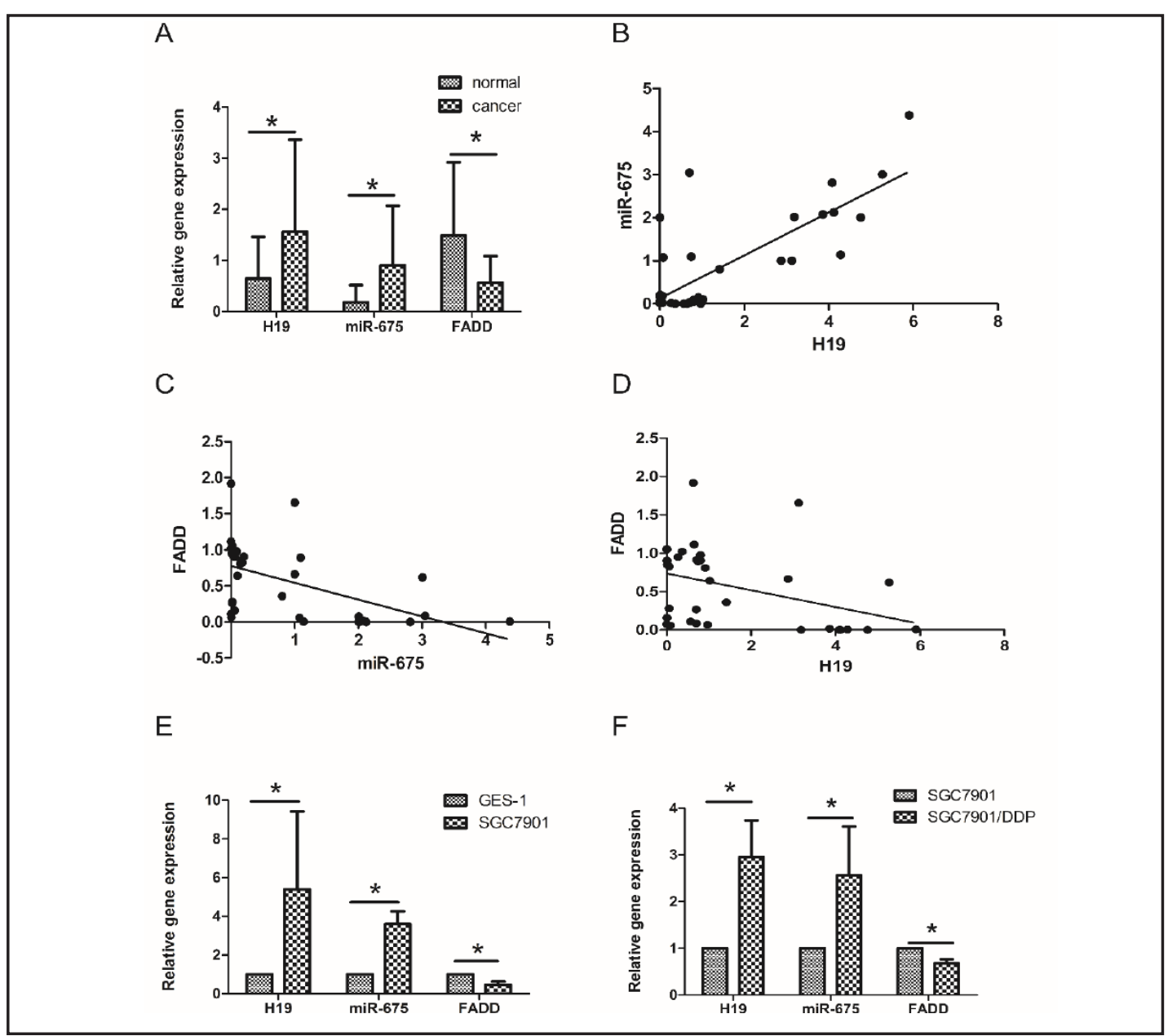

Fig. 2. Correlation of H19, miR-675 and FADD mRNA expression in gastric cancer tissues corresponding noncancerous tissues, and cell lines. (A) Relative expression of H19, miR-675, and FADD mRNA in gastric cancer tissues compared with corresponding noncancerous tissues examined by qRT-PCR. H19 and FADD mRNA expression was normalized to the GAPDH expression while miR-675 expression was normalized to U6 expression. (B) H19 and miR-675 expression was positively correlated in gastric cancer tissues. (C) Negative correlation between miR-675 and FADD expression was observed in gastric cancer tissues. (D) Negative correlation between H19 and FADD expression was observed in gastric cancer tissues. (E) H19, miR-675, and FADD mRNA expression were examined by qRT-PCR in gastric cancer cell line SGC-7901 and compared with their expression in normal gastric epithelial cell line GES-1. (F) H19, miR-675, and FADD expression were examined by qRT-PCR in cisplatin resistance gastric cancer cell line SGC-7901/DDP compared with their expression in the gastric cancer cell line SGC-7901. The data are presented as the mean \pm SD of three independent experiments. ${ }^{*} \mathrm{p}<0.05$.

Next we have examined the intensity of fluorescence which was significantly reduced after pc-H19 transfection compared with the pc-control. However, the expression was rescued when cells were co-transfected with miR-675 inhibitor. No significant variation of luciferase activity was observed for the FADD-mut transfection (Fig. 1J). Also, si-H19 transfection enhanced the relative fluorescence intensity while the co-transfection with miR675 mimic reversed this tendency. In addition, no significant variation of luciferase activity was observed when cells were transfected with FADD-mut reporter plasmid (Fig. 1K). Thus, luciferase assays revealed that miR-675 could bind to the FADD 3'UTR, causing a significant decrease in luciferase activity compared with the negative control cells. Therefore it can be concluded that H19 inhibits the expression of FADD through miR-675 targeting. 


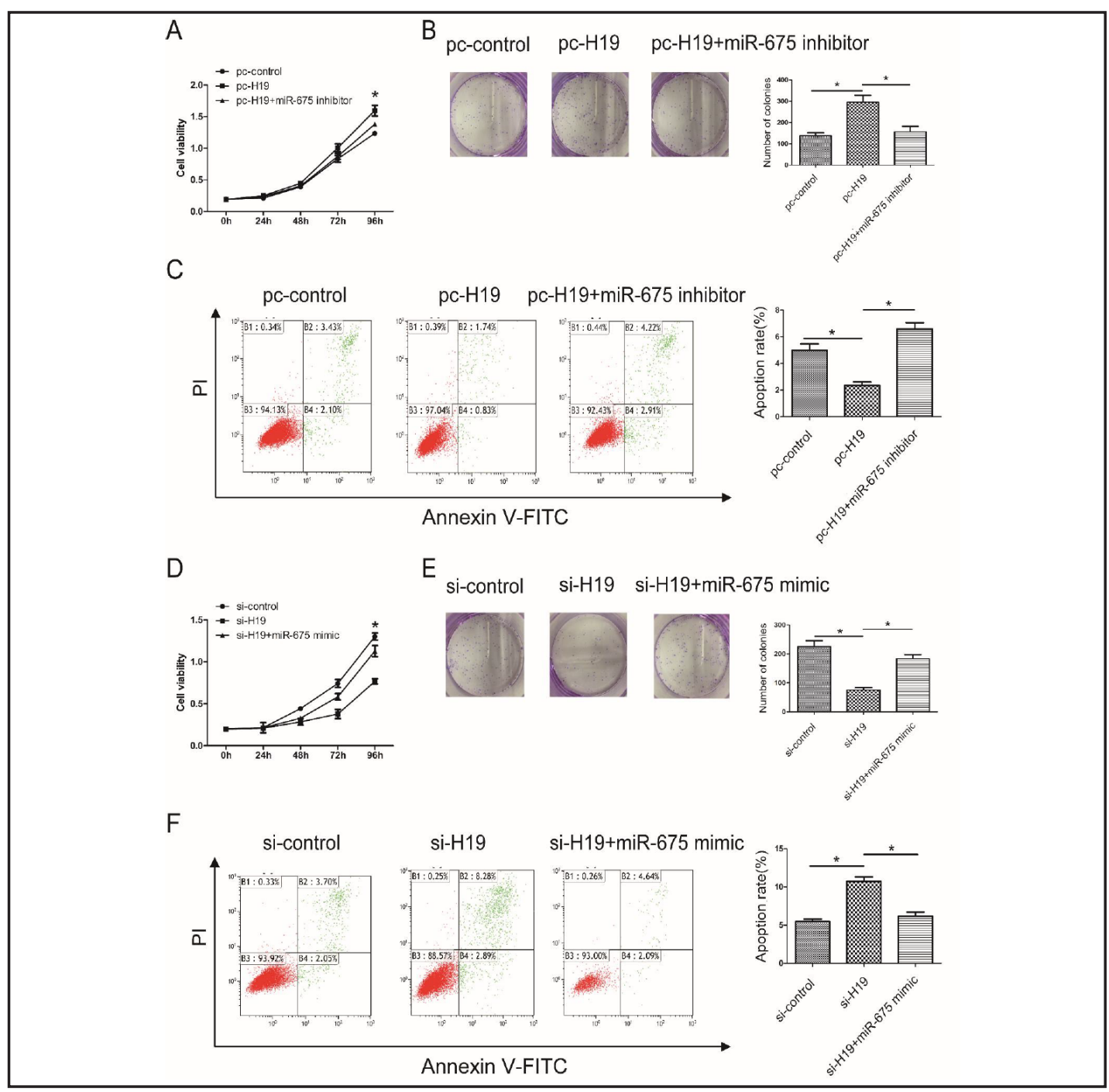

Fig. 3. MiR-675 mediates the pro-proliferation and anti-apoptosis function of H19. (A) Cell proliferation analysis using CCK-8 in SGC-7901 gastric cancer cells after their transfection with pc-control, pc-H19, or pcH19+ miR-675 inhibitor. (B) Cell proliferation analysis by measuring colony formation of SGC-7901 gastric cancer cells after their transfection with pc-control, pc-H19, or pc-H19+ miR-675 inhibitor. (C) Flow cytometry was applied to examine the apoptosis in SGC-7901 gastric cancer cells after transfection with pc-control, pc-H19, or pc-H19+ miR-675 inhibitor. (D) Cell proliferation analysis using CCK-8 in SGC-7901 gastric cancer cells after their transfection with si-control, si-H19, or si-H19+ miR-675 mimic. (E) Cell proliferation analysis by measuring colony formation of SGC-7901 after their transfection with si-control, si-H19, or siH19+ miR-675 mimic. (F) Flow cytometry was applied to examine the apoptosis in SGC-7901 gastric cancer cells after their transfection with si-control, si-H19, or si-H19+ miR-675 mimic. For all quantitative results, data are presented as the mean \pm SD of three independent experiments. ${ }^{*} \mathrm{p}<0.05$.

Correlation of H19, miR-675 and FADD mRNA expression

To further examine the correlation of H19, H19-derived miR-675 and FADD mRNA expression, we have examined their expression profiles in 34 pairs of primary gastric cancer tissues and corresponding non-tumor adjacent tissues. In our study average expression levels of both $\mathrm{H} 19$ and miR-675 were markedly up-regulated (more than two times) in tumor tissues compared to non-tumor adjacent gastric tissues. On the contrary, FADD mRNA expression was significantly decreased in tumor tissues compared to adjacent non-tumor tissue (Fig. 2A). Moreover, the H19 expression was positively correlated with miR-675 


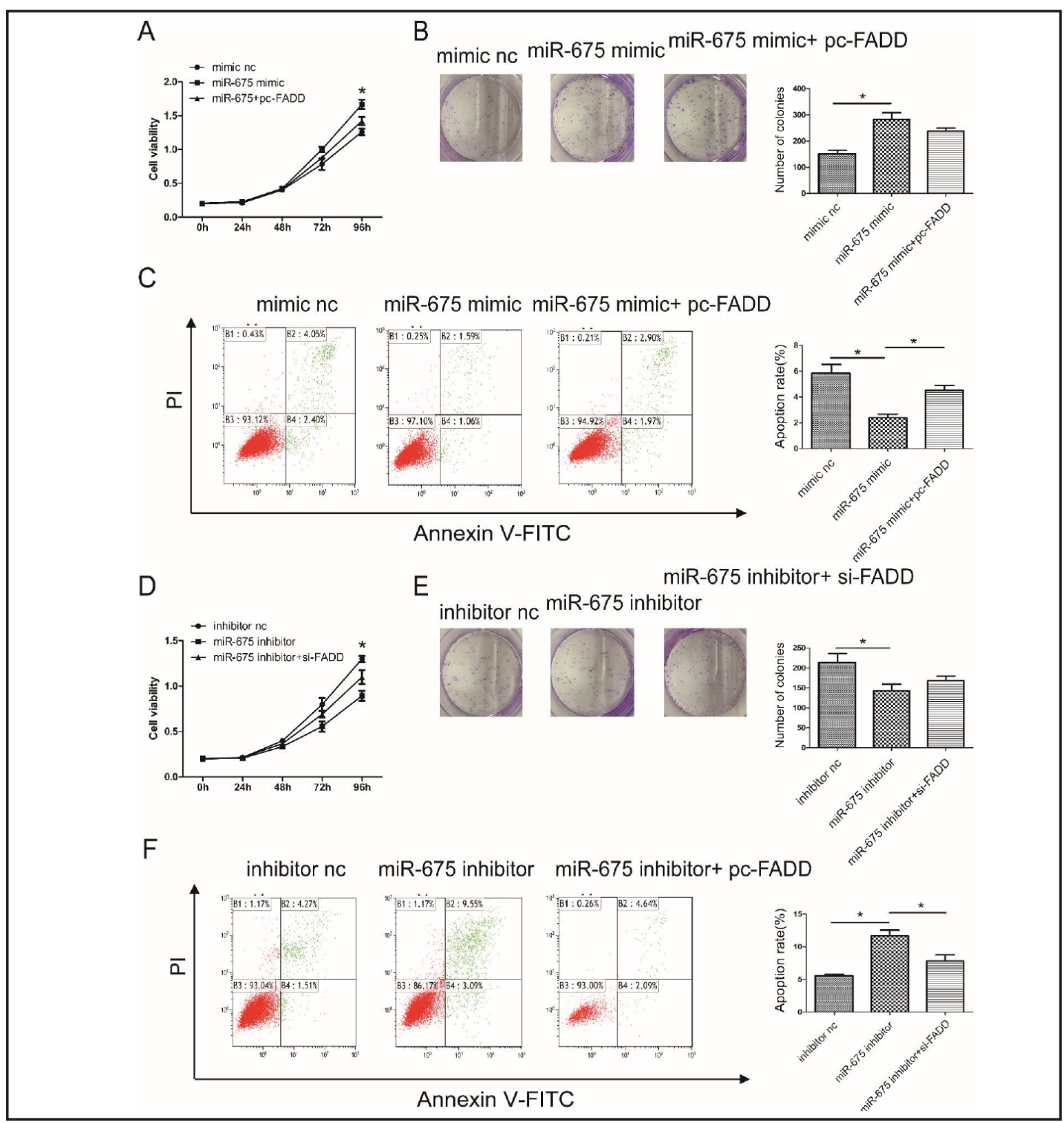

Fig. 4. FADD mediates the pro-proliferation and anti-apoptosis function of miR-675. (A) Cell proliferation was assessed by using CCK-8 in SGC-7901 gastric cancer cells after their transfection with mimic nc, miR675 mimic, or miR-675 mimic+ pc-FADD. (B) Colony formation assay was used to measure SGC-7901 cell proliferation after their transfection with mimic nc, miR-675 mimic, or miR-675 mimic+ pc-FADD. (C) Flow cytometry was applied to examine the apoptosis of SGC-7901 gastric cancer cells after their transfection with mimic nc, miR-675 mimic, or miR-675 mimic+ pc-FADD. (D) Cell proliferation was assessed by using CCK-8 in SGC-7901 gastric cancer cells after their transfection with inhibitor nc, miR-675 inhibitor, or miR675 inhibitor+ si-FADD. (E) Colony formation assay was used to measure cell proliferation in SGC-7901 gastric cancer cells after their transfection with inhibitor nc, miR-675 inhibitor, or miR-675 inhibitor+ siFADD. (F) Flow cytometry was applied to examine the apoptosis in SGC-7901 gastric cancer cells after their transfection with inhibitor nc, miR-675 inhibitor, or miR-675 inhibitor+ si-FADD. For all quantitative results, data are presented as the mean \pm SD of three independent experiments. ${ }^{*} \mathrm{p}<0.05$.

$\left(\mathrm{r}^{2}=0.5933, \mathrm{P}<0.001\right.$, Fig. 2B). The correlation analysis also revealed that FADD mRNA expression was inversely correlated with both miR-675 $\left(\mathrm{r}^{2}=0.2797, \mathrm{P}=0.0013\right.$, Fig. $\left.2 \mathrm{C}\right)$ and H19 expression $\left(r^{2}=0.1470, P=0.0252\right.$, Fig. 2D).

Next we have examined H19, miR-675, and FADD mRNA expression in normal gastric epithelial cell line GES-1, gastric cancer cell line SGC-7901, and cisplatin resistant gastric 
Fig. 5. Knockdown of H19 partially suppressed tumor growth induced by miR-675 in nude mice. (A) SGC-7901 gastric cancer cells were used for the in vivo study. Nude mice carrying tumors from respective treatment groups were shown. (B) Tumor growth curves were measured after the injection of lentivirus. Tumor volume was calculated every 4 days. (C) Tumor weight was measured after mice were euthanized. Data are presented as the mean \pm SD of three independent experiments. ${ }^{*} \mathrm{p}<0.05$.

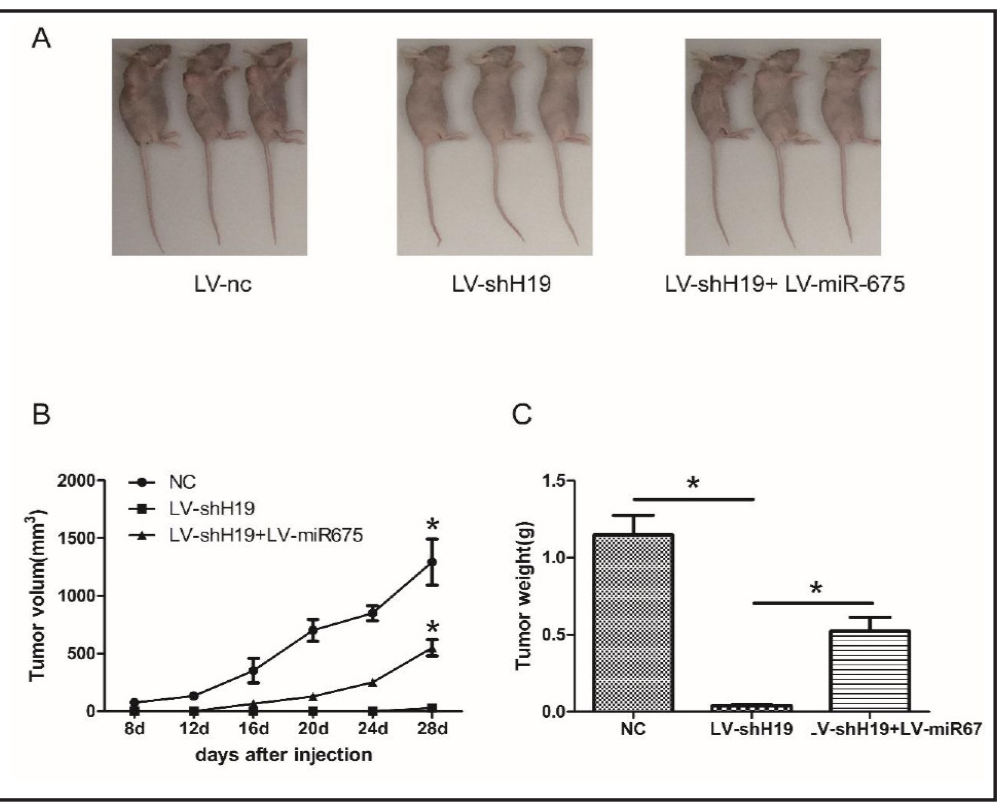

cancer cell line SGC-7901/DDP. Our results confirmed that H19 and miR-675 were highly expressed in SGC-7901 compared to GES-1, while FADD expression was lower in SGC-7901 compared to GES-1 (Fig. 2E). In addition, qRT-PCR revealed that H19 and miR-675 were overexpressed and FADD was decreased in SGC-7901/DDP, suggesting H19/ miR-675 expression may be associated with cisplatin resistance (Fig. 2F).

MiR-675 mediates the pro-proliferation and anti-apoptosis function of H19

In order to examine H19/miR-675 function and possible correlation, we have transfected SGC-7901 cells with pc-H19, si-H19, miR-675 mimic, or miR-675 inhibitor. Both CCK-8 and colony formation assay showed enhanced proliferation in H19-upregulated cells. To further investigate if $\mathrm{H} 19$ regulates cell proliferation partially by generating miR-675, we co-transfected SGC-7901 cells with pc-H19 and miR-675 inhibitor. Interestingly, the proliferation of such cells was abrogated compared to SGC-7901 cells transfected H19 (Fig. $3 \mathrm{~A}$ and B). Next, flow cytometry analysis was conducted to confirm the role of H19/miR-675 in cell apoptosis and we noticed that cell apoptosis rate was reduced in the presence of H19 compared to SGC-7901 cells transfected with scrambled control. However, the apoptosis rate was increased when cells were co-transfected with miR-675 inhibitor (Fig. 3C). In addition, we found that $\mathrm{H} 19$ downregulation could inhibit SGC-7901 cells proliferation and promote apoptosis while the miR-675 mimic could reverse these effects (Fig. 3D, E, and F). Overall, these results show that miR-675 is a functional component of the $\mathrm{H} 19$ which contributes to increased proliferation and apoptosis inhibition in SGC-7901 cells.

FADD mediates the pro-proliferation and anti-apoptosis function of miR-675

To examine the possibility whether miR-675 itself regulates gastric cancer growth by targeting FADD, we studied the phonotypes of SGC-7901cells transfected with miR-675 mimic, miR-675 inhibitor, pc-FADD, or si-FADD. In our study overexpression of miR-675 promoted gastric cancer cell proliferation and inhibited apoptosis in SGC-7901. Next, we have examined whether its target FADD was involved in this regulation. Indeed, pc-FADD co-transfection with miR-675 mimic specifically ameliorated the effect of miR-675 in gastric cancer cells (Fig. 4A, B, and C). In addition, co-transfection with si-FADD also rescued the anti-proliferative and pro-apoptotic effect of miR-675 inhibitor, thus implying that miR-675 indeed has a role in the regulation of these processes in gastric cancer by targeting FADD (Fig. 4D, E, and F). 
Fig. 6. H19/miR-675 targets FADD and inhibits caspase 8/caspase 3 . (A) Western blot analysis of FADD, pro-caspase 3 , and pro-caspase 8 expression in SGC-7901 cells transfected with pc-control, pc-H19, pc-H19+miR-675 inhibitor, si-control, si-H19, or si-H19+miR-675 mimic; An unrelated protein Actin was used as control. (B) Western blot analysis of FADD, pro-caspase 3, and pro-caspase 8 in SGC-7901 cells transfected with mimic nc, miR-675 mimic, miR-675 mimic+ pc-FADD, inhibitor nc, miR-675 inhibitor, miR675 inhibitor+ si-FADD; An unrelated protein actin was used as the control. (C) A proposed schematic model of H19/miR-675/FADD/caspase signaling pathway in gastric cancer. For all quantitative results, data are presented as the mean \pm SD of three independent experiments. $* \mathrm{p}<0.05$.

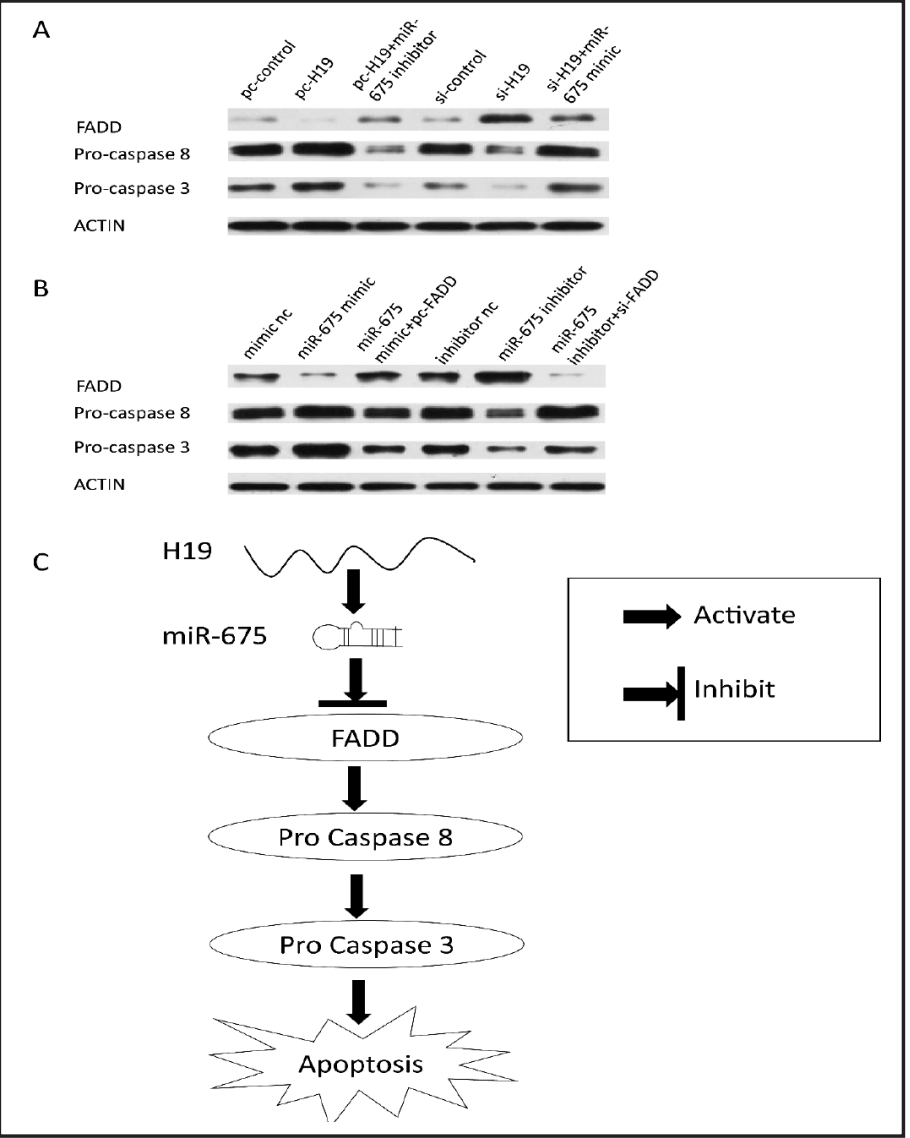

H19/miR-675 axis on gastric cancer cell growth in nude mice

To further explore the role of H19/miR-675 axis in gastric cancer in vivo, negative control infected SGC-7901 cells, LV-shH19 infected cells, and LV-shH19 and LV-miR675 coinfected cells were injected subcutaneously into nude mice. The tumor growth-curve of tumor volume was drawn, and tumor weight was measured after euthanasia. In our study there was a dramatic decrease in in tumor volume and weight in nude mice in which LVshH19 infected SGC-7901 cells were injected. In addition, co-infection with LV-miR675 partially reversed this tendency (Fig. 5A, B, C).

\section{H19/miR-675 targets FADD and inhibits caspase 8/caspase 3}

Previous studies have shown that FADD promotes apoptosis by activating the caspase pathway. To determine whether H19/miR-675 promotes cancer by inhibiting FADD and repressing the downstream caspase pathway, we assessed the expression of FADD, procaspase 8 , and pro-caspase 3 by western blot analysis. In our study expression of FADD was reduced, while expression of pro-caspase 8 and pro-caspase 3 were increased in cells overexpressing H19 or miR-675 mimic. In addition, transfection of SGC-7901 cells with si-H19 or miR-675 inhibitor had an opposite effect on their expression. Furthermore, cotransfection with pc-H19 and miR-675 inhibitor rescued the regulatory effect of pc-H19. Moreover, co-transfection with si-H19 and miR-675 mimic could alter the regulation effect of si-H19. In addition, transfection of pc-FADD could partially activate inhibited caspase signaling by miR-675 mimic and si-FADD inhibited the effect of miR-675 inhibitor (Fig. $6 \mathrm{~A}$ and B). Based on these findings we have proposed a possible mechanism of H19/miR675 action in human gastric cancer schematically in Fig. 6c. Taken together, these findings indicate that $\mathrm{H} 19$ and its derived miR-675 can inhibit caspase signaling pathway by targeting FADD. 


\section{Discussion}

Nowadays, numerous lncRNAs have been reported to play important role in carcinogenesis at both transcriptional and post-transcriptional levels, but their specific mechanisms of action still remain elusive $[5,18,19,20]$. H19 has been identified as one of the lncRNAs involved in tumorigenesis, however, its role in tumor proliferation and apoptosis has been a subject of controversy for a long time. For instance, it has been shown that $\mathrm{H} 19$ can promote gastric cancer and thyroid cancer [21,22]. On the contrary, Yu et al reported that H19 potentiated choriocarcinoma cell apoptosis [12]. In our study H19 inhibited apoptosis and promoted the proliferation of gastric cancer cells, which is consistent with other recent studies [2325]. It has been established that miR-675 derives from the firs exon of H19, and miR-675 has been reported as being at least partially responsible for the regulation of $\mathrm{H} 19$ function [26, 27]. In this study both increased proliferation and amelioration of apoptosis occurred in the presence of $\mathrm{H} 19$ and miR-675 in SGC-7901 cells. In addition, miR-675 inhibitor could rescue the pro-tumorigenesis effect of H19. Both H19 and miR-675 expression showed the same trend of up- or down-regulated in SGC-7901 cells depending upon different treatments. In the present study, we also investigated $\mathrm{H} 19$ and miR-675 expression in gastric cancer tissues and we have shown that they are significantly correlated. Collectively these findings indicate that $\mathrm{H} 19$ acts through its derived miR-675.

It has been shown previously that miR-675 regulates target genes by interacting with its 3'UTR in different types of cancers. Indeed, several miR-675 targets have been reported in cancer, such as c-Cbl and Cbl-b in breast cancer, GPR55 in lung cancer, or TGBFI in prostate cancer $[26,28,29]$. In the present study, we confirmed that FADD was a direct target of miR675 in gastric cancer. FADD is an adaptor protein which is has an important role during death receptor-mediated apoptosis, and it possesses apoptosis-independent activities, including the regulation of cell proliferation and cell-cycle [30,31]. From the Targetscan database we have identified FADD as potential target of miR-675. Moreover, in our study expression of miR-675 was inversely correlated with FADD in gastric cancer tissues. In addition, up-regulation of miR-675 significantly decreased FADD mRNA and protein levels in gastric cancer cells. Also, FADD was enhanced in gastric cancer cells with miR-675 inhibition. In our study luciferase reporter activity of wild-type 3'-UTR decreased when miR-675 was overexpressed, but this effect was reversed when mutant FADD 3'-UTR was used. Finally, transfection of gastric cancer cells with pc-FADD ameliorated the oncogenic effect of miR-675overexpression. Collectively these data support the hypothesis that FADD is a downstream mediator of miR-675 in gastric cancer. Furthermore, we found that H19 is also inversely associated with FADD expression and that H19 up-regulation can inhibit FADD expression. In addition, the luciferase reporter activity of wild-type 3'-UTR was augmented in cells with decreased H19 expression, but not in cells with mutant FADD 3'-UTR. Collectively these data indicate that H19 regulates FADD through miR-675.

It is known that FADD plays a crucial role in apoptosis, which is a form of programmed cell death occurring in multicellular organisms [32]. When TNF family death ligands (e.g., TNF, FAS, TRAIL) bind to their cognate receptors, FADD and pro-caspase 8 are recruited to form the death inducing signaling complex (DISC) [33]. Meanwhile, pro-caspase 8 undergoes auto-cleavage and converts to active caspase 8 . Through cleavage, activated caspase 8 activates downstream effector caspase $3[34,35]$. Yoo et al. have reported that expression of the FADD protein was reduced in gastric cancer and that it is correlated with the stage of gastric cancer [32]. Furthermore, downregulated caspase 3 has been associated with gastric cancer formation, and serves as potential indicator of tumor development and progression [36]. Therefore we speculated that H19/miR-675 might be involved in the regulation of gastric cancer proliferation and apoptosis through targeting FADD and then inhibiting activation of caspase 8 . In this study we have examined the expression of pro-caspase 8 and pro-caspase-3 and found that they both were activated in gastric cancer cells transfected with si-H19 or miR-675 inhibitor. Meanwhile, interference of FADD in gastric cancer cells could abrogated the caspase regulating effects of miR-675. Therefore, our data demonstrate that H19/ 
miR-675 could target FADD, increase pro-caspase 8/3, and inhibit apoptosis. In addition to H19/miR-675 axis, H19 also can regulate tumorigenesis through other mechanisms involving other $\mathrm{H} 19$ exons besides the first one $[37,38]$. Therefore the analysis of the function of H19 with mutation in the exon 1 is needed in the future to illuminate possible new mechanisms and ruling out the effect of miR-675.

In conclusion, our results demonstrate that lncRNA $\mathrm{H} 19$ and its associated miR-675 act as oncogenes by promoting proliferation and inhibiting apoptosis in gastric cancer. In addition, we have shown that FADD is a direct target of miR-675 in gastric cancer cells. Moreover, by directly targeting FADD, miR-675 inhibits the activation of caspase pathway, including caspase 8/3. Finally, identification of miR-675 and its target gene FADD in gastric cancer would help improve understanding of the molecular mechanisms underlying gastric cancer development, which might provide a wider perspective on possible avenues for gastric cancer prevention and treatment.

\section{Acknowledgements}

This work was supported by National Natural Science Foundation of China (No. 81270476 and 81470830), Jiangsu standard diagnosis and treatment research for key diseases (BE2015716), the Priority Academic Program Development of Jiangsu Higher Education Institutions (JX10231802), Science and Technology Development Projects of Suzhou (SYS201304), Jiangsu postgraduate scientific research and innovation projects (KYZZ15_0265), and International Cooperation and Exchanges Projects of Nanjing Medical University.

\section{Disclosure Statement}

The authors declare no conflicts of interest.

\section{References}

1 Torre LA, Bray F, Siegel RL, Ferlay J, Lortet-Tieulent J, Jemal A: Global cancer statistics, 2012. CA Cancer J Clin 2015;65:87-108.

2 Amieva M, Peek RM Jr: Pathobiology of helicobacter pylori-induced gastric cancer. Gastroenterology 2016;150:64-78.

-3 Hartgrink HH, Jansen EP, van Grieken NC, van de Velde: CJ: Gastric cancer. Lancet 2009;374:477-490.

4 Ulitsky I, Bartel DP: lincRNAs: genomics, evolution, and mechanisms. Cell 2013;154:26-46.

-5 Yu B, Ye X, Du Q, Zhu B, Zhai Q, Li XX: The long non-coding RNA CRNDE promotes colorectal carcinoma progression by competitively binding miR-217 with TCF7L2 and enhancing the Wnt/beta-Catenin signaling pathway. Cell Physiol Biochem 2017;41:2489-2502.

6 Xiang Y, Zhang Y, Tang Y, Li Q: MALAT1 modulates TGF-beta1-induced endothelial-to-mesenchymal transition through downregulation of miR-145. Cell Physiol Biochem 2017;42:357-372.

-7 Yan J, Dang Y, Liu S, Zhang Y, Zhang G: LncRNA HOTAIR promotes cisplatin resistance in gastric cancer by targeting miR-126 to activate the PI3K/AKT/MRP1 genes. Tumour Biol 2016;37:16345-16355.

8 Zhao J, Liu Y, Zhang W, Zhou Z, Wu J, Cui P: Long non-coding RNA Linc00152 is involved in cell cycle arrest, apoptosis, epithelial to mesenchymal transition, cell migration and invasion in gastric cancer. Cell cycle 2015;14:3112-3123.

-9 Gabory A, Jammes H, Dandolo L: The H19 locus: role of an imprinted non-coding RNA in growth and development. Bioessays 2010;32:473-480.

10 Yan L, Zhou J, Gao Y, Ghazal S, Lu L, Bellone S: Regulation of tumor cell migration and invasion by the H19/ let-7 axis is antagonized by metformin-induced DNA methylation. Oncogene 2015;34:3076-3084.

11 Hao Y, Wang G, Lin C, Li D, Ji Z, Gao F: Valproic acid induces decreased expression of H19 promoting cell apoptosis in A549 cells. DNA Cell Biol 2017;36:428-435.

12 Yu LL, Chang K, Lu LS, Zhao D, Han J, Zheng YR: Lentivirus-mediated RNA interference targeting the H19 gene inhibits cell proliferation and apoptosis in human choriocarcinoma cell line JAR. BMC Cell Biol 2013;14:26. 


\section{Cellular Physiology Cell Physiol Biochem 2017;42:2364-2376 \begin{tabular}{ll|l} 
DOI: 10.1159/000480028 & O 2017 The Author(s). Published by S. Karger AG, Basel \\
www.karger.com/cpb
\end{tabular}}

Yan et al.: H19/miR-675 Promote Gastric Cancer via FADD

13 Ebert MS, Sharp PA: Roles for microRNAs in conferring robustness to biological processes. Cell 2012;149:515-524.

-14 Huntzinger E, Izaurralde E: Gene silencing by microRNAs: contributions of translational repression and mRNA decay. Nat Rev Genet 2011;12:99-110.

-15 Cai X, Cullen BR: The imprinted H19 noncoding RNA is a primary microRNA precursor. RNA 2007;13:313316.

16 Tsang WP, Ng EK, Ng SS, Jin H, Yu J, Sung JJ: Oncofetal H19-derived miR-675 regulates tumor suppressor RB in human colorectal cancer. Carcinogenesis 2010;31:350-358.

17 Keniry A, Oxley D, Monnier P, Kyba M, Dandolo L, Smits G: The H19 lincRNA is a developmental reservoir of miR-675 that suppresses growth and Igf1r. Nat Cell Biol 2012;14:659-665.

$\checkmark 18$ Esteller M: Non-coding RNAs in human disease. Nat Rev Genet 2011;12:861-874.

19 Lu X, Zhou C, Li R, Deng Y, Zhao L, Zhai W: Long noncoding RNA AFAP1-AS1 promoted tumor growth and invasion in cholangiocarcinoma. Cell Physiol Biochem 2017;42:222-230.

20 Li C, Chen J, Zhang K, Feng B, Wang R, Chen L: Progress and prospects of long noncoding RNAs (lncRNAs) in hepatocellular carcinoma. Cell Physiol Biochem 2015;36:423-434.

21 Li H, Yu B, Li J, Su L, Yan M, Zhu Z: Overexpression of IncRNA H19 enhances carcinogenesis and metastasis of gastric cancer. Oncotarget 2014;5:2318-2329.

22 Liu L, Yang J, Zhu X, Li D, Lv Z, Zhang X: Long noncoding RNA H19 competitively binds miR-17-5p to regulate YES1 expression in thyroid cancer. FEBS J 2016;283:2326-2339.

-23 Zhang EB, Han L, Yin DD, Kong R, De W, Chen J: c-Myc-induced, long, noncoding H19 affects cell proliferation and predicts a poor prognosis in patients with gastric cancer. Med Oncol 2014;31:914

24 Liu G, Xiang T, Wu QF, Wang WX: Curcumin suppresses the proliferation of gastric cancer cells by downregulating H19. Oncol Lett 2016;12:5156-5162.

25 Wang SH, Ma F, Tang ZH, Wu XC, Cai Q Zhang MD: Long non-coding RNA H19 regulates FOXM1 expression by competitively binding endogenous miR-342-3p in gallbladder cancer. J Exp Clin Cancer Res 2016;35:160.

-26 Zhu M, Chen Q Liu X, Sun Q, Zhao X, Deng R: lncRNA H19/miR-675 axis represses prostate cancer metastasis by targeting TGFBI. FEBS J 2014;281:3766-3775.

27 Liu L, An X, Li Z, Song Y, Li L, Zuo S: The H19 long noncoding RNA is a novel negative regulator of cardiomyocyte hypertrophy. Cardiovasc Res 2016;111:56-65.

28 Vennin C, Spruyt N, Dahmani F, Julien S, Bertucci F, Finetti P: H19 non coding RNA-derived miR-675 enhances tumorigenesis and metastasis of breast cancer cells by downregulating c-Cbl and Cbl-b. Oncotarget 2015;6:29209-29223.

29 He D, Wang J, Zhang C, Shan B, Deng X, Li B: Down-regulation of miR-675-5p contributes to tumor progression and development by targeting pro-tumorigenic GPR55 in non-small cell lung cancer. Mol Cancer 2015;14:73.

30 Blander JM: A long-awaited merger of the pathways mediating host defence and programmed cell death. Nat Rev Immunol 2014;14:601-618.

- 31 Kersse K, Verspurten J, Vanden Berghe T, Vandenabeele P: The death-fold superfamily of homotypic interaction motifs. Trends Biochem Sci 2011;36:541-552.

-32 Subramanian S, Steer CJ: MicroRNAs as gatekeepers of apoptosis. J Cell Physiol 2010;223:289-298.

33 Rahman M, Pumphrey JG, Lipkowitz S: The TRAIL to targeted therapy of breast cancer. Adv Cancer Res 2009;103:43-73.

-34 Kischkel FC, Hellbardt S, Behrmann I, Germer M, Pawlita M, Krammer PH: Cytotoxicity-dependent APO-1 (Fas/CD95)-associated proteins form a death-inducing signaling complex (DISC) with the receptor. EMBO J 1995;14:5579-5588.

35 Mirakian R, Nye K, Palazzo FF, Goode AW, Hammond LJ: Methods for detecting apoptosis in thyroid diseases. J Immunol Methods 2002;265:161-175.

36 Yoo NJ, Lee SH, Jeong EG, Lee JW, Soung YH, Nam SW: Expression of nuclear and cytoplasmic phosphorylated FADD in gastric cancers. Pathol Res Pract 2007;203:73-78.

37 Wang SH, Ma F, Tang ZH, Wu XC, Cai Q Zhang MD: Long non-coding RNA H19 regulates FOXM1 expression by competitively binding endogenous miR-342-3p in gallbladder cancer. J Exp Clin Cancer Res 2016;35:160-172.

-38 Zhou X, Ye F, Yin C, Zhuang Y, Yue G, Zhang G: The Interaction between MiR-141 and lncRNA-H19 in regulating cell proliferation and migration in gastric cancer. Cell Physiol Biochem 2015;36:1440-52. 